\title{
THE COMPLETION OF A RING WITH A VALUATION
}

\author{
HELEN E. ADAMS
}

\begin{abstract}
This paper proves three main results: the completion of a commutative ring with respect to a Manis valuation is an integral domain; a necessary and sufficient condition is given that the completion be a field; and the completion is a field when the valuation is Harrison and the value group is archimedean ordered.
\end{abstract}

1. Introduction. In [1] we defined a generalized pseudovaluation on a ring $R$ and found explicitly the completion of $R$ with respect to the topology induced on $R$ by such a pseudovaluation. Using this inverse limit characterization of the completion of $R$ we show, in $\S 2$, that when the codomain of a valuation on $R$ is totally ordered, the completion of $R$ with respect to the valuation has no (nonzero) divisors of zero and the valuation on $R$ can be extended to a valuation on the completion. In $\S 3, R$ is assumed to have an identity and so, from [1, $\$ 2]$, the completion has an identity: a necessary and sufficient condition is given such that each element of the completion of $R$ has a right inverse.

$\S \S 2$ and 3 are immediately applicable to a Manis valuation $\varphi$ on a commutative ring $R$ with identity [3], where the valuation $\varphi$ is surjective and the set $\varphi(R)$ is a totally ordered group. A Harrison valuation on $R$ is a Manis valuation $\varphi$ on $R$ such that the set $\{x: x \in R, \varphi(x)>\varphi(1)\}$ is a finite Harrison prime [2]. In $\S 4$ we show that the completion of a ring with respect to a Harrison valuation is a field if the value group is archimedean ordered.

Let $R$ be a ring (not necessarily either commutative or with identity). Let $S$ be a totally ordered quasi-residuated $[1, \S 1]$ semigroup and let $S_{0}$ be the disjoint union of $S$ and a zero element $0_{S}$ with the properties: $0_{S} 0_{S}=$ $0_{S}$; and, for any $s \in S, 0_{S}>s$ and $s 0_{S}=0_{S}=0_{S} s$. Let $\varphi: R \rightarrow S_{0}$ be a valuation on $R$. That is, for all $a, b \in R$,

(1.1) $\varphi(a b)=\varphi(a) \varphi(b)$;

(1.2) $\varphi(a-b) \geqq \min \{\varphi(a), \varphi(b)\}$;

(1.3) $\varphi(0)=0_{S}$;

(1.4) the set $\varphi(R) \backslash\left\{0_{S}\right\}$ is nonempty.

Received by the editors October 19, 1971.

AMS 1970 subject classfications. Primary 12J20, 13J10; Secondary 16A80.

Key words and phrases. Valuation on a ring, valuated ring completion, Manis valuation, Harrison prime.

(C) American Mathematical Society 1972 
Note that, if $\varphi(a) \neq \varphi(b)$, then

$$
\varphi(a-b)=\min \{\varphi(a), \varphi(b)\} .
$$

Now if $S$ has a greatest element $\pi$, it is clear from [1] that the completion of $R$ with respect to $\varphi$ is isomorphic to the ring $R /\{x: x \in R, \varphi(x) \geqq \pi\}$. Hence let $S$ have no greatest element.

Following [1], let $\left\{P_{s}\right\}_{s \in S}$ be the generalized filtration defined on $R$ as follows: For each $s \in S, P_{s}=\{x: x \in R, \varphi(x) \geqq s\}$. Note that, in this case, $\bigcup_{s \in S} P_{s}=R$. From [1, §2], the completion of $R$ with respect to $\varphi$ is the topological ring $R=$ proj $\lim R / P_{s}$, with zero $\mathbf{0}=\left\{P_{s}\right\}_{s \in S}$, and addition, multiplication and topology defined in [1, §2]. For reference, $R=$ $\left\{\left\{\xi_{s}\right\}_{s \in S} \in \prod_{s \in S} R / P_{s}\right.$ : for all $s, t \in S$ such that $\left.s \geqq t, \xi_{s} \subseteq \xi_{t}\right\}$. When there is no risk of ambiguity, $\left\{\xi_{s}\right\}_{s \in S}$ will be written as $\left\{\xi_{s}\right\}$.

\section{2. $\boldsymbol{R}$ has no divisors of zero. First we need}

LEMmA 1. Let $\left\{\xi_{s}\right\}_{s \in S} \in \boldsymbol{R} \backslash\{\boldsymbol{0}\}$. Then there exist $u, v \in S$ with $u<v$ such that $\xi_{u}=P_{u}, \xi_{v} \neq P_{v}$, and, for all $x, y \in \xi_{v}, u \leqq \varphi(x)=\varphi(y)<v$.

Proof. (i) Let $s \in S, x \in \xi_{s}$. Since $\bigcup_{s \in S} P_{s}=R$, there exists $t \in S$ such that $x \in P_{t}$. If $t \geqq s$, then $x \in P_{s}$ from [1, (1.2)], and so $\xi_{s}=P_{s}$. Otherwise $t<s, \xi_{s} \subseteq \xi_{t}$, and so $\xi_{t}=x+P_{t}=P_{t}$. Hence there exists $u \in S$ such that $\xi_{u}=P_{u}$.

(ii) Since $\left\{\xi_{s}\right\}_{s \in S} \neq \mathbf{0}$, there exists $v \in S$ such that $\xi_{v} \neq P_{v}$. Now if $v \leqq u$, then $P_{u}=\xi_{u} \subseteq \xi_{v}$, and so $\xi_{v}=P_{v}$. Therefore $v>u$.

(iii) Now $\varphi(x)<v$ for all $x \in \xi_{v}$, since otherwise $\xi_{v}=P_{v}$. Since $v>u$, $\xi_{v} \subseteq \xi_{u}=P_{u}$ and so $\varphi(x) \geqq u$ for all $x \in \xi_{v}$. Suppose there exist $y, z \in \xi_{v}$ such that $\varphi(y) \neq \varphi(z)$. Then $\min \{\varphi(y), \varphi(z)\}=\varphi(y-z) \geqq v$ since $y-z \in P_{v}$, which contradicts $\varphi(y), \varphi(z)<v$. Hence $\varphi(x)=\varphi(y)$ for all $x, y \in \xi_{v}$. This completes the proof.

THEOREM 1. $\boldsymbol{R}$ has no divisors of zero.

Proof. Let $\left\{\xi_{s}\right\},\left\{\eta_{s}\right\} \in \boldsymbol{R}$ such that $\left\{\xi_{s}\right\}\left\{\eta_{s}\right\}=\mathbf{0}$. Suppose that $\left\{\xi_{s}\right\}$, $\left\{\eta_{s}\right\} \neq 0$. Then, by Lemma 1 , there exist $a, b, t, u \in S$ such that, for all $x \in \xi_{t}$, $\varphi(x)=a$; and, for all $y \in \eta_{u}, \varphi(y)=b$.

Since $S$ has no maximal element, there exists $c \in S$ such that $c>a b$; and, by the definition of multiplication in $R[1, \S 2]$, there exists $v \in S$ such that, for all $x \in \xi_{v}$ and for all $y \in \eta_{v}, x y \in P_{c}$.

Let $w \geqq t, u, v$. Then, for all $x \in \xi_{w} \subseteq \xi_{t}$ and for all $y \in \eta_{w} \subseteq \eta_{u}, \varphi(x y)=$ $a b<c$, which contradicts $x y \in P_{c}$.

Hence $\left\{\xi_{s}\right\}=0$ or $\left\{\eta_{s}\right\}=0$ which completes the proof. 
COROLlaRy. The completion of a commutative ring with respect to a nontrivial Manis valuation is an integral domain.

Lemma 1 also enables us to define a valuation on $\boldsymbol{R}$. Let $\left\{\xi_{s}\right\} \in \boldsymbol{R} \backslash\{\boldsymbol{0}\}$. Then there exists $t \in S$ such that $\xi_{t} \neq P_{t}$. Define $\varphi\left(\left\{\xi_{s}\right\}\right)=\varphi(x)$ where $x \in \xi_{t}$. Define $\varphi(0)=0_{S}$. Then it is a straightforward task to show that $\varphi: R \rightarrow S_{0}$ is a well defined valuation on $\boldsymbol{R}$. Moreover, the valuation $\varphi$ on $\boldsymbol{R}$ is an "extension" of the valuation $\varphi$ on $R$ in the sense that $\varphi$ restricted to the image $i(R)$ of $R$ in $R$, defined in [1, Theorem 2.1], satisfies $\varphi(i(x))=$ $\varphi(x)$ for each $x \in R$. And it is easy to show that $\varphi$ defines the topology $\mathscr{T}$ $[1, \S 2]$ on $R$.

3. When elements of the completion have inverses. Let $R$ have identity 1. Then, from [1, Proposition 2.1], $R$ has identity $\left\{1+P_{s}\right\}_{s \in S}$.

Proposition 1. Each element of $\boldsymbol{R}$ has a right inverse if and only if (1) given $a \in R$ such that $\varphi(a) \neq 0_{S}$, and given $s \in S$, there exists $b \in R$ such that $\varphi(a b-1) \geqq s$.

Proof. (i) Let each element of $R$ have a right inverse. Let $a \in R$ be such that $\varphi(a) \neq 0_{S}$, and let $w \in S$. Clearly, $\left\{a+P_{s}\right\} \neq 0$ since $S$ has no maximal element. Hence there exists $\left\{\eta_{s}\right\} \in \boldsymbol{R}$ such that $\left\{a+P_{s}\right\}\left\{\eta_{s}\right\}=$ $\left\{1+P_{s}\right\}$. By the definition of multiplication in $R$, there exists $t \in S$ such that, for all $x \in \eta_{t}, a x \in 1+P_{w}$. Hence (1) holds.

(ii) Conversely, let (1) hold. Denote $\varphi(1)$ by $1_{S}$. Let $\left\{\xi_{s}\right\} \in \boldsymbol{R} \backslash\{0\}$. By Lemma 1 there exist $\rho, \sigma \in S$ such that $\xi_{\rho} \neq P_{\rho}$ and, for all $x \in \xi_{\rho}, \varphi(x)=\sigma$; $\sigma$ is a constant for the chosen $\left\{\xi_{s}\right\}$.

For each $s \in S$, let $x_{s} \in \xi_{s}$ and let $y_{s} \in R$ be such that $\varphi\left(x_{s} y_{s}-1\right)>\sigma s(S$ has no maximal element); let $m_{s} \in S$ be such that $m_{s}>\sigma s$ and let $g(s) \in S$ be such that $g(s) \geqq m_{s} \sigma, s$. Let $t \in S$ be such that $\sigma t>1_{S}$ and $t \geqq \rho$. Then, for each $s \in S$ such that $s \geqq t, \sigma \varphi\left(y_{s}\right)=\varphi\left(x_{s} y_{s}\right)=1_{s}$. Define, for each $s \in S$,

$$
\begin{aligned}
\eta_{s} & =y_{g(s)}+P_{s} \quad \text { if } s \geqq t, \\
& =y_{g(t)}+P_{s} \quad \text { if } s<t .
\end{aligned}
$$

We shall show that $\left\{\xi_{s}\right\}\left\{\eta_{s}\right\}=\left\{1+P_{s}\right\}$.

(a) We must show that, for each $s \in S, \eta_{s}$ is well defined. Accordingly, for each $s \in S$, let $w_{s} \in \xi_{s}, z_{s} \in R$ and $\tau, u, n_{s}, h(s) \in S$ be such that $\xi_{r} \neq P_{r}$; $\varphi\left(w_{s} z_{s}-1\right)>\sigma s ; n_{s}>\sigma s$ and $h(s) \geqq n_{s} \sigma, s ; \sigma u>1_{S}$ and $u \geqq \tau ;$ and

$$
\begin{aligned}
\zeta_{s} & =z_{h(s)}+P_{s} \quad \text { if } s \geqq u, \\
& =z_{h(u)}+P_{s} \quad \text { if } s<u .
\end{aligned}
$$

We must show that, for each $s \in S, \zeta_{s}=\eta_{s}$.

Claim. Let $a, b, c \in S$ be such that $a, b \geqq c \geqq t$. Then $z_{h(a)}-y_{g(b)} \in P_{c}$. 
SUBPROOF. We shall refer to $h(a)$ and $g(b)$ as $h$ and $g$ respectively. Now

$$
w_{h}\left(z_{h}-y_{g}\right)=\left(w_{h} z_{h}-x_{g} y_{g}\right)+\left(x_{g}-w_{h}\right) y_{g} .
$$

Clearly, $\varphi\left\{w_{h} z_{h}-1-\left(x_{g} y_{g}-1\right)\right\}>\sigma c$. Also $\varphi\left(x_{g}-w_{h}\right) \geqq r \sigma$ where $r=$ $\min \left\{m_{b}, n_{a}\right\}>\sigma c$. Hence $\varphi\left\{\left(x_{g}-w_{h}\right) y_{g}\right\}>\sigma c$ since $\sigma \varphi\left(y_{g}\right)=1_{S}$, and so $\varphi\left\{w_{h}\left(z_{h}-y_{g}\right)\right\}>\sigma c$. Thus $\varphi\left(z_{h}-y_{g}\right) \geqq c$ since $\varphi\left(w_{h}\right)=\sigma$. This proves the claim.

Let $s \in S$. Without loss of generality, let $u \geqq t$. Then, by applying the claim to each of the cases $s \geqq u, u \geqq s \geqq t, t>s$, we see that $\zeta_{s}=\eta_{s}$. Thus $\eta_{s}$ is well defined for each $s \in S$.

(b) By further application of the claim, it is easy to show that $\left\{\eta_{s}\right\}_{s \in S} \in \boldsymbol{R}$.

(c) Finally we must show that $\left\{\xi_{s}\right\}\left\{\eta_{s}\right\}=\left\{1+P_{s}\right\}$. Let $\left\{\xi_{s}\right\}\left\{\eta_{s}\right\}=\left\{\Omega_{s}\right\}$. Let $s \in S$. Then there exists $v \in S$ such that $x y \in \Omega_{s}$ for all $x \in \xi_{v}, y \in \eta_{v}$. Let $d \in S$ be such that $\sigma d \geqq s$ and $d \geqq v, t$. Then $x_{g(d)} \in \xi_{v}, y_{g(d)} \in \eta_{v}$ and $\varphi\left(x_{g(d)} y_{g(d)}-1\right) \geqq s$. Hence $\Omega_{s}=x_{g(d)} y_{g(d)}+P_{s}=1+P_{s}$ which completes the proof of the proposition.

COROllary. Let $\varphi: R \rightarrow S_{0}$ be a nontrivial Manis valuation on a commutative ring $R$ with identity 1 . Then the completion of $R$ with respect to $\varphi$ is a field if and only if condition (1) holds.

4. Harrison valuations. In [2, pp. 11 and 12 and Proposition 2.2, p. 14], Harrison shows that, given a finite prime $P$ of a commutative ring $R$ with identity, one can define a Manis valuation $\varphi_{P}: R \rightarrow \Gamma_{P}$ on $R$ such that the set $\left\{x: x \in R, \varphi_{P^{\prime}}(x)>\varphi_{P}(1)\right\}=P$. Using the corollary to Proposition 1 , we have:

THEOREM 2. Let $\varphi: R \rightarrow S_{0}$ be a nontrivial Harrison valuation on a commutative ring $R$ with identity $1_{R}$, such that $S$ be archimedean ordered. Then the completion of $R$ with respect to $\varphi$ is a field.

Proof. Let $a \in R$ be such that $\varphi(a) \neq 0_{S}$ and let $s \in S$. Now $\{x: x \in R$, $\left.\varphi(x)>1_{S}\right\}=P$, a finite Harrison prime of $R$. Suppose $a R \subseteq P$. Then, for all $x \in R, \varphi(a) \varphi(x)>1_{S}$, which contradicts $\varphi(a) \neq 0_{S}$ since $\varphi$ is surjective and $S$ is a totally ordered group. Hence $a R+P$ properly contains $P$. But $a R+P$ is closed under addition and multiplication. Thus $-1_{R} \in a R+P$, since $P$ is a Harrison prime: that is, there exist $c \in R, d \in P$ such that $a c-1_{R}=d$.

Since $\varphi(d)>1_{S}$ and $S$ is archimedean ordered, there exists a natural number $m$ such that $\varphi\left(d^{m}\right)=\{\varphi(d)\}^{m} \geqq s$. Let $n$ be an odd integer such that $n \geqq m$. Then $\varphi\left(d^{n}\right) \geqq s$. Now $d^{n}=\left(a c-1_{R}\right)^{n}=a b-1_{R}$ where $b \in R$. The theorem then follows from Proposition 1. 


\section{REFERENCES}

1. Helen E. Adams, Filtrations and valuations on rings, Pacific J. Math. 41 (1972).

2. D. K. Harrison, Finite and infinite primes for rings and fields, Mem. Amer. Math. Soc. No. 68 (1966), 62 pp. MR 34 \#7550.

3. Merle E. Manis, Valuations on a commutative ring, Proc. Amer. Math. Soc. 20 (1969), 193-198. MR 38 \#2134.

Department of Mathematics, Mount Holyoke College, South Hadley, MasSACHUSETTS 01075 